\title{
Processamento Visual da Forma em Idosos: Curvas de Limiar de Contraste para Freqüências Angulares e Senoidais
}

\author{
Natanael Antonio dos Santos \\ Universidade Federal da Paraiba \\ Maria Lúcia de Bustamante Simas \\ Renata Maria Toscano Barreto Lyra Nogueira \\ Universidade Federal de Pernambuco
}

\begin{abstract}
Resumo
O objetivo deste trabalho foi mensurar curvas de sensibilidade ao contraste para freqüências angulares e senoidais em idosos. Foram estimados limiares de contraste para um grupo de jovens com idade entre 20-26 anos e um grupo de idosos com idade entre 60-67 anos. Todos os sujeitos apresentavam acuidade visual normal e se encontravam livres de doenças oculares identificáveis. As freqüências angulares e senoidais estimadas com o método psicofísico da escolha forçada foram: 2, 4, 8, 16, 24, 32, 48, 64 e 96 ciclos $/ 360^{\circ}$ e $0.5,1,2,3,4,6$ e $9 \mathrm{cpg}$, respectivamente. Os resultados mostraram declínio na sensibilidade ao contraste nos idosos nas freqüências angulares e senoidais médias e altas comparados aos jovens. Encontramos, ainda, que a sensibilidade ao contraste dos idosos nas freqüências angulares baixas é melhor do que a dos jovens. Estes resultados são consistentes com alterações na sensibilidade ao contraste relacionados ao processo de envelhecimento.

Palavras-chave: Processamento visual da forma; freqüência angular; freqüência espacial; sensibilidade ao contraste, método da escolha forçada.
\end{abstract}

Visual Processing of Form in the Aged: Threshold Contrast Curves to Angular and Spatial Frequency

\begin{abstract}
The aim of this work was to measure contrast sensitivity curves for angular and spatial frequencies in the aged. We measured the contrast thresholds from young adults (20-26 years-old) and aged (60-67 years-old). All the participants had good visual acuity and were free from significant ocular pathology. The angular and spatial frequencies measured with a forced-choice psychophysic method were $2,4,8,16,24,32,48,64$ and 96 cycle $/ 360^{\circ}$ and $0.5,1,2,3,4,6$ and $9 \mathrm{cpd}$, respectively. The results showed a loss in contrast sensitivity for the aged at high and medium angular and spatial frequencies compared to the younger group. We still found that the contrast sensitivity of the aged group at low angular frequency is better than the younger group. These results are consistent with age-related changes in contrast sensitivity function.

Keywords: Visual processing of form; angular frequency; spatial frequency; contrast sensitivity; forced-choice method.
\end{abstract}

Estudos psicofísicos em humanos e estudos neurofisiológicos em macacos e gatos fornecem forte suporte para a idéia de que o processamento visual de objetos é composto por mecanismos ou canais múltiplos sintonizados para bandas estreitas de freqüências espaciais (R. L. De Valois \& K. K. De Valois, 1988; Santos, 1999; Santos \& Simas, 2001a; Simas \& Santos, 2002; Wilson, Levi, Maffei, Rovamo \& De Valois, 1990). O modelo de canais múltiplos assume que a função de sensibilidade ao contraste (FSC) é o envelope da sensibilidade para a série total de canais, cada um sensível a uma região do espectro (Braddick, Campbell \& Atkinson, 1978; Graham \& Nachmias, 1971). Neste contexto, a FSC tem sido utilizada para descrever e compreender os mecanismos putativos que medeiam o processamento visual da forma e de detalhes espaciais. A

${ }^{1}$ Endereço para correspondência: Rua Manoel de Macedo, s/n, 401, Ed Camburiú, 58052 290. João Pessoa, PB. Fone: (81) 32713734, Fax: (81) 32718271. E-mail: natanael@npd.ufpe.br sensibilidade ao contraste é definida como a recíproca da quantidade mínima de contraste necessária para detectar um padrão qualquer (Ex.: uma grade) de uma determinada freqüência espacial (Cornsweet, 1970; Woodhouse \& Barlow, 1982). Assim, o contraste para cada freqüência espacial é ajustado com um procedimento comportamental ou psicofísico até que o sistema visual possa discriminar um padrão (ou objeto) de freqüência espacial de um outro com um campo homogêneo de luminância média. Em resumo, a sensibilidade ao contraste estima a visibilidade de qualquer objeto em função de sua freqüência espacial.

A FSC de humanos com funções visuais normais tem formato geral bem definido. Isto é, apresenta sensibilidade máxima nas freqüências intermediárias ou médias, aproximadamente no centro da curva, com atenuações nas freqüências baixas e altas, nos extremos da curva.

Freqüência espacial é o número de ciclos (listras claras e escuras) por unidade de espaço, que em percepção visual da forma foi convencionalmente denominado de ciclo por 
grau de ângulo visual (cpg). Por exemplo, uma freqüência espacial de 2 cpg teria duas listras claras e duas escuras por grau de ângulo visual. Enquanto, o contraste é definido pela relação entre a luminância máxima (listra clara) e mínima (listra escura) dividida pela soma da luminância máxima e mínima.

\section{A Sensibilidade ao Contraste e o Envelhecimento Humano}

A existência de alterações significativas nas características neurais e ópticas do sistema visual com a idade originaram inicialmente vários estudos tentando relacionar o processo de envelhecimento à sensibilidade ao contraste, ou ainda, avaliar o desempenho do sistema visual humano em faixas etárias diferentes (Arden, 1978; Arden \& Jacobson, 1978; McGrath \& Morrison, 1981; Owsley, Sekuler \& Siemsen, 1983; Sekuler, Hutman \& Owsley, 1980; Skalka, 1980). Alguns destes trabalhos, conforme veremos a seguir, merecem destaque particular porque apresentam resultados divergentes. Por exemplo, Arden (1978) mensurou a FSC para freqüências espaciais baixas e médias utilizando sujeitos na faixa etária de 11 a 70 anos. Neste estudo, o autor utilizou placas fotográficas onde cada placa continha uma freqüência espacial específica e o contraste variava de um nível subliminar, na parte superior, a um nível supra-liminar, na parte inferior da placa. Arden mensurou o limiar de contraste para cada freqüência investigada utilizando um procedimento no qual o investigador descobria lentamente a placa até o sujeito detectar o estímulo, ou seja, a grade. Os resultados de Arden não revelaram mudanças na sensibilidade ao contraste relacionadas ao processo de envelhecimento em nenhuma freqüência espacial testada.

Por outro lado, Arden e Jacobsen (1978), utilizando o mesmo aparato, procedimento e amostra semelhante, encontraram sensibilidade levemente reduzida para os voluntários mais velhos em todas as freqüências espaciais testadas. Resultados semelhantes foram encontrados por Skalka (1980) e por McGrath e Morrison (1981). Enquanto, Skalka utilizou as placas de Arden (1978), McGrath e Morrison utilizaram um osciloscópio com luminância média de $2 \mathrm{~cd} / \mathrm{m}^{2}$ para apresentar os estímulos.

Por sua vez, Sekuler e colaboradores (1980) mensuraram a sensibilidade ao contraste para dois grupos de voluntários com idade média de 18 e 73 anos. O limiar para cada grade foi mensurado com o método psicofísico do ajustamento. As freqüências espaciais foram apresentadas em um monitor com contraste controlado por um microcomputador. Os resultados encontrados mostram sensibilidade menor para freqüências espaciais baixas e médias no grupo de idosos comparado ao grupo de jovens. Os autores não encontraram diferenças entre o grupo de jovens e idosos para a freqüência mais alta, $16 \mathrm{cpg}$.

Outros, contrários aos estudos citados acima, relatam que o envelhecimento afeta principalmente a sensibilidade de freqüências espaciais mais altas (Arundale, 1978; Derefeldt, Lennerstrand \& Lundh, 1979). Derefeldt e colaboradores mensuraram a FSC de adultos jovens e idosos. Os limiares foram estimados com grades senoidais apresentadas em um osciloscópio. Os resultados demonstraram que os voluntários com idade acima de 60 anos apresentaram sensibilidade reduzida para freqüências espaciais acima de 4 cpg quando comparado aos grupos mais jovens.

Finalmente, alguns trabalhos relatam que $o$ envelhecimento afeta principalmente as freqüências espaciais médias e altas (Crassini, Brown \& Bowman, 1988; Elliott, 1987; Owsley \& cols., 1983; Ross, Clarke \& Bron, 1985). Por exemplo, Owsley e colaboradores (1983), em estudo detalhado, mensuraram a FSC de voluntários na faixa etária de 19 a 87 anos, utilizando freqüências espaciais de $0,5,1,2$, 4, 8 e 16 cpg. Os voluntários foram divididos em sete grupos de idade (19-28, 31-38, 41-48, 50-58, 60-69, 70-79 e 80-87 anos). Os resultados mostraram que a sensibilidade ao contraste para freqüências espaciais baixas (Ex.: 0,5 e 1 cpg) permanece a mesma em todos os grupos de idade. Por outro lado, a sensibilidade ao contraste para freqüências espaciais médias e altas (2-16 cpg) diminui com a idade a partir da faixa etária 41-48 anos. Este declínio torna-se mais severo com o aumento da idade, de forma que o grupo de voluntários acima de 60 anos apresenta perdas estatisticamente significantes na sensibilidade ao contraste para as freqüências espaciais médias e altas comparado ao grupo mais jovem.

No momento, não existe ainda um consenso geral sobre as faixas de freqüências que são afetadas pelo envelhecimento, entretanto a idéia principal é que o processo de envelhecimento reduz principalmente a sensibilidade ao contraste nas freqüências espaciais médias e altas (Fiorentini, Porciatti, Morrone \& Burr, 1996; Schefrin, Tregear, Harvey Jr. \& Werner, 1999).

\section{O Presente Estudo}

Declínio na sensibilidade ao contraste em humanos, depois dos 50 anos, tem sido demonstrado por estudos psicofísicos utilizando grade senoidal vertical ou freqüências espaciais como estímulos visuais (Nio \& cols., 2000). Porém, pesquisas envolvendo padrões com outras configurações, como por exemplo freqüências angulares, são dispersas e raras com adultos jovens e não encontramos nada com 
idosos. Neste sentido, o presente estudo teve como objetivo mensurar a curva de limiar de contraste para freqüências angulares e a curva de limiar de contraste para freqüências espaciais para duas faixas etárias diferentes (adultos jovens de 20-26 anos e idosos de 60-67 anos) utilizando o método psicofísico da escolha forçada.

\section{Método}

\section{Participantes}

Participaram dos experimentos 10 voluntários, 5 adultos jovens com idade entre 20-26 anos e 5 idosos com idade entre 60-67 anos. Todos os participantes estavam livres de doenças oculares identificáveis e a menos de 12 meses tinham consultado os seus oftalmologistas. Os mesmos também apresentavam acuidade visual normal ou corrigida.

\section{Equipamentos e Estímulos}

Foi utilizado um sistema que compreende um monitor de vídeo SONY-BVM-1910, com entrada "RGBsync" controlado por um microcomputador através de um placa Data-Translation DT-2853. Um programa escrito em linguagem $C$ foi desenvolvido no laboratório para executar os experimentos. Uma cadeira foi fixada a 150 $\mathrm{cm}$ da tela do monitor de vídeo. Uma tábua cinza foi situada acima do monitor de vídeo para onde o voluntário, entre uma apresentação e outra, deveria fixar o olhar com o objetivo de diminuir os efeitos de pós-imagem. A luminância média foi de $6,9 \mathrm{~cd} / \mathrm{m}^{2}$ ajustada por um fotômetro do tipo SPOT METTER, com precisão de um grau, ASAHI PENTAX. A luminância mínima foi de $6,2 \mathrm{~cd} / \mathrm{m}^{2}$ e a máxima de $7,5 \mathrm{~cd} / \mathrm{m}^{2}$. O ambiente do laboratório era cinza para melhor controle da luminância.

Os estímulos de freqüências espaciais e angulares foram gerados em tons de cinza e apresentados em tempo real no monitor. Todos os estímulos também eram circulares com um diâmetro de 7,25 graus de ângulo visual a $150 \mathrm{~cm}$ de distância da tela (Figura 1).

As estimativas foram feitas utilizando pares de estímulos, estímulo de teste e estímulo de fundo. O estímulo de teste foi uma das freqüências espaciais $0,5,1,2,3,4,6$ ou $9 \mathrm{cpg}$ (sete condições experimentais) ou uma das freqüências angulares $2,4,8,16,24,32,48,64$ e 96 ciclos $/ 360^{\circ}$ (nove condições experimentais), enquanto o estímulo de fundo foi sempre um padrão homogêneo com luminância média de $6,9 \mathrm{~cd} / \mathrm{m}^{2}$. A Figura 1 ilustra exemplos de freqüências espaciais e angulares. Mais informações sobre estes estímulos podem ser encontrados em Santos e Simas (Santos, 1999; Santos \& Simas, 2001b; Simas, Santos \& Thiers, 1997; Simas \& Santos, 2002).
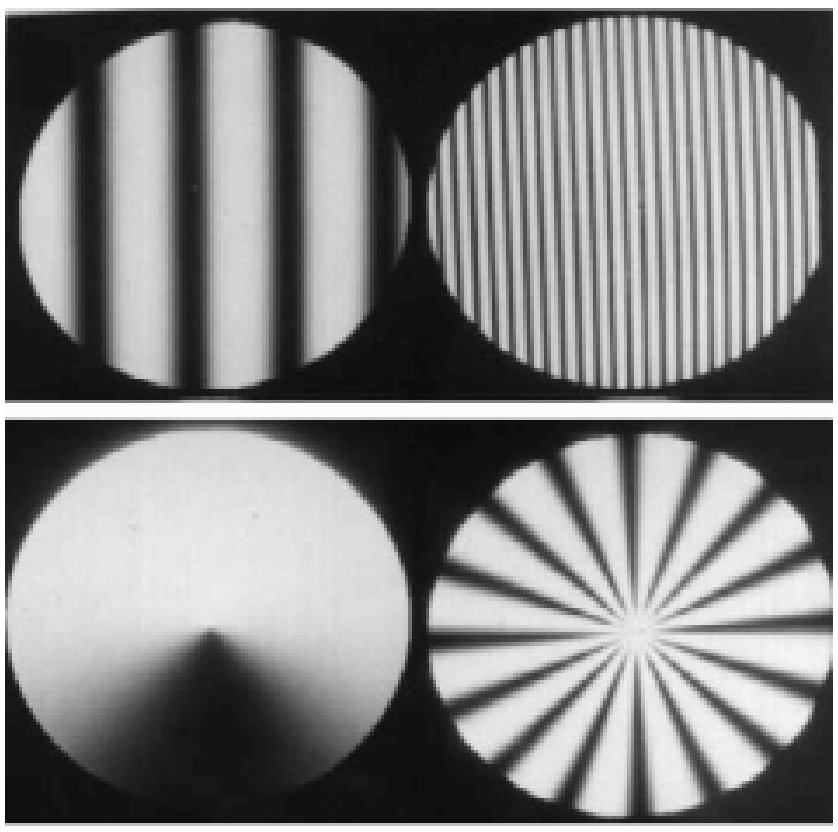

Figura 1. Exemplos de estímulos de freqüências espaciais grades senoidais (acima à esquerda $0,5 \mathrm{cpg}$ e à direita $3 \mathrm{cpg}$ ) e freqüências angulares (embaixo à direita 1 ciclo $/ 360^{\circ}$ e à esquerda 16 ciclos $/ 360^{\circ}$ ). Estímulos originalmente calibrados para serem vistos a $150 \mathrm{~cm}$ de distância antes de fotografados.

\section{Procedimento}

As estimativas foram realizadas com método da escolha forçada (Santos, 1999; Simas \& cols., 1997). Este método foi baseado no estudo de Wetherill e Levitt (1965), que calcula a probabilidade de acertos consecutivos por parte do voluntário, ou seja, em cerca de 100-150 apresentações de escolhas entre os dois estímulos, o estímulo de teste é percebido $79 \%$ das vezes pelo voluntário. O procedimento para medir o limiar para cada freqüência consistiu na apresentação sucessiva simples do par de estímulos e o voluntário teria que escolher dentre eles qual continha a freqüência espacial ou angular. O critério adotado para variar o contraste da freqüência de teste (espacial ou angular) era o de três acertos consecutivos para decrescer uma unidade e um erro para acrescer da mesma unidade $(0,08 \%)$.

Durante cada sessão experimental era apresentada uma seqüência de estímulos que foi iniciada com um sinal sonoro seguido imediatamente pela apresentação do primeiro estímulo por $2 \mathrm{~s}$, seguido de um intervalo entre estímulos de $1 \mathrm{~s}$, seguido pela apresentação do segundo estímulo por $2 \mathrm{~s}$ e da resposta do voluntário. A ordem de apresentação dos estímulos era aleatória. Se a resposta do voluntário fosse correta, era seguida por outro sinal sonoro e um intervalo de $3 \mathrm{~s}$ para a seqüência se repetir. Em outras palavras, o intervalo entre tentativas era de $3 \mathrm{~s}$ 
independente da resposta (ou escolha) ser correta ou não. O sinal sonoro que indica o início da apresentação do par de estímulos e o que indicava a escolha correta eram diferentes e discretos. A sessão experimental variava em duração dependendo dos erros e acertos do voluntário até proporcionarem um total de 10 máximos e 10 mínimos para os adultos jovens (ou cinco máximos e cinco mínimos para os idosos) conforme requerido para o final automático da mesma.

Cada um dos pontos (ou freqüências) da curva de limiar de contraste foi estimado pelo menos duas vezes, em dias diferentes, por cada um dos observadores. No total, 10 curvas foram medidas para cada grupo de voluntários, gerando uma amostra de aproximadamente 120 valores para cada um dos pontos estimados. Todas as estimativas foram medidas à distância de $150 \mathrm{~cm}$, com visão binocular.

Os voluntários foram orientados antes da sessão a pressionar a barra de espaço quando julgassem que o estímulo de teste tivesse sido apresentado primeiro e qualquer tecla acima da barra de espaço quando julgasse que o mesmo tivesse sido apresentado em segundo lugar, isto é, após o estímulo de fundo. Em outras palavras, a tarefa do observador foi escolher sempre o estímulo que continha a freqüência espacial ou angular.

\section{Resultados}

As Figuras 2 e 3 mostram as curvas de limiares de contraste de adultos jovens e de idosos para freqüências espaciais e para freqüências angulares, respectivamente. As freqüências espaciais e angulares são apresentadas nos gráficos em função da quantidade de contraste mínimo necessário para detectar cada freqüência. As barras verticais indicam as barras de erro-padrão da média.

Nosso tratamento estatístico foi estimar a barra de erro-padrão da média para cada distribuição de 120 valores mensurados para cada ponto e corrigidos para o tamanho da amostra pelo estatístico t-student para obter o nível de confiança de 99\%. Em experimentos anteriores, foi estabelecido que a barra de erro-padrão da média, corrigida pelo tamanho da amostra representando intervalo de confiança de $99 \%$ pelo estatístico t-student, de acordo com o número de valores mensurados, é um critério mais estrito do que a utilização da ANOVA ou teste t para amostras correlacionadas. Por exemplo, quando os intervalos dos erros-padrão da média assim corrigidos se superpõem até mesmo pela metade, ainda assim um teste $t$-student para amostras correlacionadas revela diferenças entre as médias que são significativas com $p<0,05$. Nos casos em que os erros barras não se sobrepõem, as médias são significativas $\operatorname{com} p<0,001$. A
ANOVA tende a mostrar interações e efeitos significativos em todos fatores e não acrescenta muita informação.

A curva de limiar de contraste (1/FSC) é a recíproca da função de sensibilidade ao contraste (FSC). Em outras palavras, quanto menor o limiar de contraste maior a sensibilidade do sistema visual humano e vice-versa. Assim, os menores valores de limiares correspondem aos maiores valores de sensibilidade ao contraste.

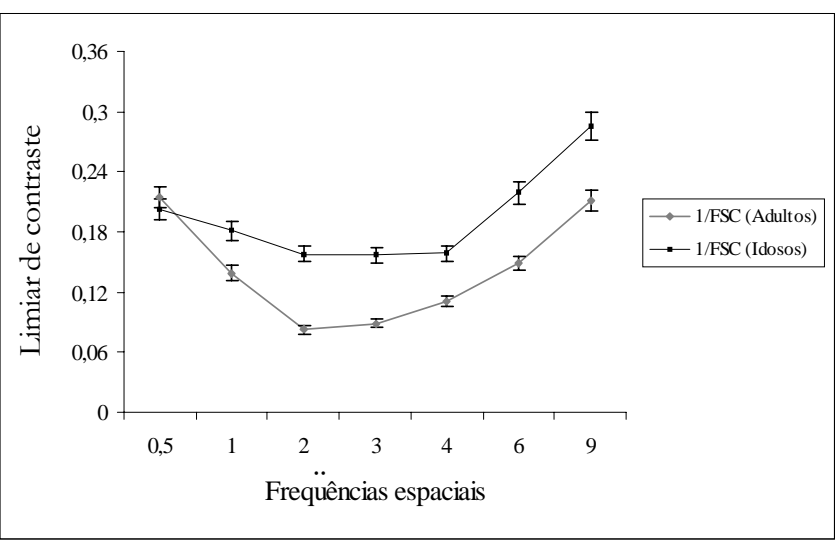

Figura 2. Curva de limiar de contraste de adultos e idosos para freqüências espaciais.

A Figura 2 mostra que a sensibilidade máxima dos dois grupos ocorreu aproximadamente na mesma faixa de freqüências espaciais, isto é, entre 2-3 cpg no grupo de jovens e entre 2-4 cpg no grupo de idosos. Entretanto, o limiar de contraste do grupo de idosos foi de aproximadamente duas vezes mais alto do que o grupo de jovens na faixa de máxima sensibilidade. A Figura 2 mostra, ainda, que o limiar de contraste do grupo de idosos nas freqüências mais altas $(6 \mathrm{e}$ 9 cpg) é aproximadamente 1,5 mais alto do que o limiar do grupo de jovens.

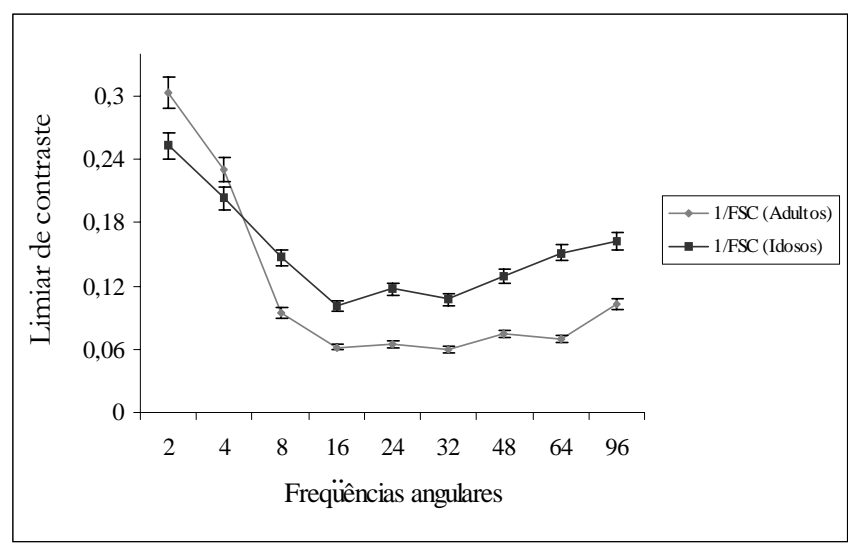

Figura 3. Curva de limiar de contraste de adultos e idosos para freqüências angulares. 
A Figura 3 mostra que a sensibilidade máxima dos dois grupos ocorreu na mesma faixa de freqüência angular, isto é, entre 16-32 ciclos. Entretanto, o limiar de contraste do grupo de idosos foi da ordem de 1,7 vezes mais alto do que o limiar de contraste do grupo de jovens tanto nas freqüências angulares médias (faixa de máxima sensibilidade) quanto nas freqüências angulares mais altas comparado ao grupo de adultos jovens. Ao contrário, nas freqüências angulares baixas (Ex.: 2 ciclos) a situação se inverte, ou seja, o limiar de contraste do grupo de jovens foi aproximadamente 1,2 vezes mais alto do que o limiar de contraste do grupo de idosos. O mesmo não ocorreu para freqüências espaciais baixas (ver Figura 2).

Os nossos resultados são consistentes com o declínio da sensibilidade ao contraste do sistema visual do idoso apenas para freqüências espaciais e angulares médias e altas. Não encontramos declínio na sensibilidade do grupo de idosos nas freqüências espaciais e angulares baixas. No caso específico das freqüências angulares baixas (Ex.: 2 e 4 ciclos), o grupo de idosos apresentou melhor sensibilidade do que o grupo de adultos jovens. Estes resultados demonstram que de certa forma o sistema visual do idoso funciona melhor nas freqüências baixas do que nas médias e altas.

\section{Discussão}

Declínio na sensibilidade ao contraste relacionado ao envelhecimento tem sido demonstrado por vários estudos psicofísicos conforme discutimos no início deste trabalho. Embora, existam divergências sobre as faixas de freqüências espaciais que são afetadas pelo processo de envelhecimento. Entretanto, é importante ressaltar que questões metodológicas e o estado de saúde da amostra podem ser responsáveis por boa parte das divergências encontradas. Por exemplo, estudos realizados com as placas de Arden podem ser influenciados pela iluminação e pela velocidade com que cada grade é descoberta pelo experimentador (Owsley \& cols., 1983). Assim como, já se sabe que doenças comuns da terceira idade (glaucoma, catarata, diabetes, dentre outras) afetam diferentemente a sensibilidade ao contraste (Woods, Tregear \& Mitchell, 2001). Não está claro em alguns experimentos discutidos acima se todos os voluntários apresentavam boa acuidade visual e estavam livres de doenças ou patologias oculares significantes.

Qual a contribuição dos fatores ópticos ou neurais na redução da sensibilidade ao contraste espacial no idoso? Esta questão ainda é objeto de investigação (McLellan, Marcos \& Burns, 2001; Nio \& cols., 2000; Schefrin \& cols., 1999). Entretanto, em condições fotópicas, tanto alterações senis nas propriedades ópticas do olho (Ex.: miose senil, opacidade da córnea) quanto os mecanismos neurais responsáveis pelo processamento visual da forma podem estar relacionados à deterioração da sensibilidade ao contraste para freqüências espaciais altas, enquanto que os fatores neurais parecem exercer um papel mais proeminente no declínio da sensibilidade para freqüências espaciais abaixo de $2 \mathrm{cpg}$ (Schefrin \& cols., 1999).

A nossa proposta principal foi investigar o efeito da idade na curva de sensibilidade ao contraste para freqüências angulares, pois já existe na literatura um número considerável de estudos relacionando limiar de contraste, idade e freqüência espacial. Os nossos resultados com freqüência espacial e angular, nas mesmas condições e método, demonstram declínio na sensibilidade ao contraste no grupo de idosos nas freqüências espaciais e angulares médias e altas (Figuras 2 e 3). Os resultados com grade senoidal apresentados na Figura 2 são semelhantes a outros relatados na literatura que não encontraram alteração relacionada ao envelhecimento nas freqüências espaciais baixas, e sim, declínio na sensibilidade ao contraste apenas nas freqüências espaciais médias e altas dos idosos (Crassini \& cols., 1988; Elliott, 1987; Owsley \& cols., 1983; Ross \& cols., 1985). Já os resultados apresentados na Figura 3 para freqüências angulares são semelhantes aos da Figura 2 apenas nas freqüências médias e altas. Pois, nas freqüências angulares baixas, houve um aumento na sensibilidade do grupo de idosos quando comparado ao grupo de jovens. Este achado não seria necessariamente o esperado, até porque não encontramos nada parecido na literatura para freqüências espaciais ou grade senoidal vertical. Entretanto, estímulos de freqüência espacial (grade senoidal) e estímulos angulares são diferentes. Grade senoidal é um estímulo elementar cuja luminância varia senoidalmente no espaço, em uma direção, em um sistema de coordenadas cartesianas (Figura 1). Enquanto, o estímulo de freqüência angular é um padrão cuja luminância varia, de acordo com o seno ou o co-seno, na direção angular em um sistema de coordenadas polares. Neste caso, a fase da freqüência angular varia entre 0 (zero) e $2 \mathrm{p}$ e a freqüência é discreta e sempre dada por número inteiro de ciclos por $360^{\circ}$ (Figura 1). Assim, ao contrário da grade senoidal, o estímulo angular é adimensional, inteiro e sua freqüência independe da distância.

Será que o processo de envelhecimento afeta diferentemente o sistema visual? Será que os mecanismos que processam estímulos cartesianos (Ex.: grades senoidais) são mais afetados pelo envelhecimento do que os que processam estímulos polares (Ex.: freqüências angulares)? Esses questionamentos são preliminares já que não encontramos nada na literatura nesta direção. Entretanto, estudos psicofísicos em humanos e neurofisiológicos em animais (gato e primata) mostram que estímulos lineares em coordenadas cartesianas (Ex.: freqüência espacial ou 
grade senoidal) e estímulos em coordenadas polares (Ex.: freqüência angular e radial) são processados por áreas visuais distintas (Desimone \& Schein, 1987; R. L. De Valois \& K. K. De Valois, 1988; Gallant, Brau \& van Essen, 1993; Gallant, Connor, Rakshit, Lewis, \& van Essen, 1996; Manahilov \& Simpson, 2001; Merigan, 1996; Wilkinson \& cols., 2000; Wilson \& Wilkinson, 1998; Wilson, Wilkinson \& Asaad, 1997). Em outras palavras, freqüências espaciais seriam filtradas no córtex visual primário ou estriado (V1), enquanto freqüências angulares em áreas visuais extra-estriadas (Ex.: área visual V4 e córtex visual ínfero temporal, IT). Estes achados, também, são reforçados com a técnica de imagem de ressonância magnética funcional em humanos (Wilkinson \& cols., 2000) que demonstra inicialmente que estímulos radiais e angulares em coordenadas polares são processados em áreas do córtex extra-estriado (V4 e IT). No momento, não podemos afirmar que o envelhecimento afeta diferentemente o processamento visual de freqüências espaciais e angulares.

Estes resultados envolvendo sensibilidade ao contraste, idosos e freqüências angulares são preliminares. Novas pesquisas estão sendo conduzidas para estudar de forma mais abrangente e sistemática os efeitos do envelhecimento na sensibilidade ao contraste angular.

\section{Referências}

Arden, G. B. (1978). The importance of measuring contrast sensitivity in cases of visual disturbance. British Journal of Ophthalmology, 62, 198-209.

Arden, G. B. \& Jacobsen, J. (1978). A simple grating test for contrast sensitivity: Preliminary results indicate value for screening in glaucoma. Investigative Ophthalmology \& Visual Science, 17, 23-32.

Arundale, K. (1978). An investigation into the variation of human contrast sensitivity with age and ocular pathology. British Journal of Ophthalmology, 62, 213-215.

Braddick, F. W., Campbell, F. W. \& Atkinson, J. (1978). Channels in vision: Basic aspects. Em R. Held, H. W. Leibowitz \& H. L. Teuber (Orgs.), Handbook of sensory physiology: Perception (Vol. V, pp. 3-38). New York: Springer-Verlag.

Cornsweet, T. N. (1970). Vision perception. New York: Academic Press.

Crassini, B., Brown, B. \& Bowman, D. (1988). Age-related changes in contrast sensitivity in central and peripheral retina. Perception, 17, 315332.

Derefeldt, F. D., Lennerstrand, G. \& Lundh, B. (1979). Age variations in normal human contrast sensitivity. Acta Ophthalmology, 57, 679-690.

Desimone, R. \& Schein, S. J. (1987). Visual properties of neurons in area V4 of macaque: Sensitivity to stimulus form. Journal of Neurophysiology, 57, 835-867.

De Valois, R. L. \& De Valois, K. K. (1988). Spatial vision. New York: Oxford University Press.

Elliott, D. B. (1987). Contrast sensitivity decline with ageing: A neural or optical phenomenon? Opbthalmic and Physiological Optics, 7, 415-419.

Fiorentini, A., Porciatti, V., Morrone, M. C. \& Burr, D. C. (1996). Visual ageing: Unspecific decline of the responses to luminance and colour. Vision Research, 36, 3557-3566.

Gallant, J. L., Brau, J. \& van Essen, D. C. (1993). Selectivity for polar hyperbolic, and cartesian gratings in macaque visual cortex. Science, 259, 100-103.
Gallant, J. L., Connor, C. E., Rakshit, S., Lewis, J. W. \& van Essen, D. C. (1996). Neural responses to polar, hyperbolic, and cartesian gratings in area V4 of the macaque monkey. Journal of Neurophysiology, 76, 27182739.

Graham, N. \& Nachmias, J. (1971). Detection of grating patterns containing two spatial frequencies: A comparison of single-cannel and multiple channel models. Vision Research, 11, 251-259.

Manahilov, V. \& Simpson, W. A. (2001). Energy model for contrast detection: Spatial-frequency and orientation selectivity in grating summation. Vision Research, 41, 1547-1560.

McGrath, C. \& Morrison, J. D. (1981). The effects of age on spatial frequency perception in human subjects. Quarterly Journal of Experimental Physiology, 66, 253-261.

McLellan, J. S., Marcos, S. \& Burns, S. A. (2001). Age-related changes in monochromatic wave aberrations of the human eye. Investigative Ophthalmology \& Visual Science, 42, 1390-1395.

Merigan, W. H. (1996). Basic visual capabilities and shape discrimination after lesions of extrastriate area V4 in macaques. Visual Neuroscience, 13, 51-60.

Nio, Y. K., Jansonius, N. M., Fidler, V. Geraghty, E., Norrby, S. \& Kooijman, A. C. (2000). Age-related changes of defocus-specific contrast sensitivity in healthy subjects. Ophthalmic and Physiological Optics, 20, 323-334.

Owsley, C., Sekuler, R. \& Siemsen, D. (1983). Contrast sensitivity throughout adulthood. Vision Research, 23, 689-699.

Ross, J. E., Clarke, D. D. \& Bron, A. J. (1985). Effect of age on contrast sensitivity function: Uniocular and binocular findings. British Journal of Ophthalmology, 69, 51-56.

Santos, N. A. (1999). Sistema visual humano: Curvas de sensibilidade e filtragem de frequiências angulares, radiais e radiall angulares acopladas. Tese de Doutorado não-publicada, Curso de Pós-Graduação em Neurociências e Comportamento, Instituto de Psicologia, Universidade de São Paulo. São Paulo, SP.

Santos, N. A. \& Simas, M. L. B. (2001a). Percepção e processamento visual da forma: Discutindo modelos teóricos atuais. Psicologia: Reflexãod Crítica, 14, 157-166.

Santos, N. A. \& Simas, M. L. B. (2001b). Função de sensibilidade ao contraste: Indicador da percepção visual da forma e da resolução espacial. Psicologia: Reflexãod Crítica, 14, 589-597.

Schefrin, B. E., Tregear, S. J., Harvey Jr., L. O. \& Werner, J. S. (1999). Senescent changes in scotopic contrast sensitivity. Vision Research, 39 , 3728-3736.

Sekuler, R., Hutman, L. P. \& Owsley, C. (1980). Human aging and spatial vision. Science, 209, 1255-1256.

Simas, M. L. S. \& Santos, N. A. (2002). Narrow-band 1, 2, 3, 4, 8, 16 and 24 cycles $/ 360^{\circ}$ angular frequency filters. Brazilian Journal of Medical and Biological Research, 35, 243-253.

Simas, M. L. S., Santos, N. A. \& Thiers, F. A. (1997). Contrast sensitivity to angular frequency stimuli is higher than that for sine wave gratings in the respective middle range. Brazilian Journal of Medical and Biological Research, 30, 633-636.

Skalka, H. W. (1980). Effect of age on Arden grating acuity. British Journal of Ophthalmology, 64, 21-23.

Wetherill, G. B. \& Levitt, H. (1965). Sequential estimation of points on a psychometric function. The British Journal of Mathematical and Statistical Psychology, 48, 1-10.

Wilkinson, F., James, T. W., Wilson, H. R., Gati, J. S., Menon, E. S. \& Goodale, M. A. (2000). An fMRI study of the selective activation of human extrastriate form vision areas by radial and concentric gratings. Current Biology, 10, 1455-1458. 
Wilson, H. R., Levi, D., Maffei, L., Rovamo, J. \& De Valois, R. (1990). The perception of form: Retina to striate cortex. Em S. W. Spillmann \& J. S. Werner (Orgs.), Visual perception: The neurophysiological foundation (pp. 231-271). New York: Academic Press.

Wilson, H. R. \& Wilkinson, F. (1998). Detection of global structure in glass patterns: Implications for form vision. Vision Research, 38, 29332947.

Wilson, R. W., Wilkinson, F. \& Asaad, W. (1997). Concentric orientation summation in human form vision. Vision Research, 37, 2325-2330.

Woodhouse, J. M. \& Barlow, H. B. (1982). Spatial and temporal resolution and analysis. Em H. B. Barlow \& J. D. Mollon (Orgs.), The senses (pp. 133-164). Cambridge University Press.
Woods, R. L., Tregear, S. J. \& Mitchell, R. A. (1997). Screening for ophthalmic disease in older subjects using visual acuity and contrast sensitivity. Ophthalmology, 105, 2318-2326.

Sobre os Autores

Natanael Antonio dos Santos é Doutor em Psicologia (Neurociências e Comportamento) pelo Instituto de Psicologia Experimental da Universidade de São Paulo (1999). Professor Adjunto do Departamento de Psicologia da Universidade Federal da Paraíba. Coordenador do Laboratório de Processamento Visual - LabPVis. Linha de Pesquisa: Percepção e Processamento Visual da Forma em Humanos.

Maria Lúcia de Bustamante Simas é Doutora em Psicologia pela Queen's University, Ontario, Canada (1985). Professora Adjunta do Departamento de Psicologia da Universidade Federal de Pernambuco. Coordenadora do Laboratório de Percepção Visual - LabVis. Bolsista do CNPq. Linha de Pesquisa: Percepção e Processamento Visual da Forma.

Renata Maria Toscano Barreto Lyra Nogueira é Graduanda de Psicologia da Universidade Federal de Pernambuco. Bolsista de Iniciação Científica, CNPq, vinculada ao LabVis-UFPE. 